\title{
Prof. Dr. Franz Ruttner zum siebzigsten Geburtstag
}

Unter den Naturforschern, die in massgeblicher Weise der modernen Limnologie den Weg wiesen, ist Franz RutTNER als einer der ersten zu nennen. Der siebzigste Geburtstag, den er am I2. Mai I952 feiern konnte, mag uns Anlass sein, diesem bedeutenden österreichischen Kollegen auch von der Schweiz aus ein Wort der hohen Verehrung und des herzlichen Dankes auszusprechen.

Einer grossen Zahl von Hydrobiologen aus aller Welt ist es ergangen wie dem Verfasser dieser Zeilen: ein Aufenthalt in Franz RutTNers Biologischer Station am Lunzer Untersee (Niederösterreich) hat genügt, um sie für ein Leben lang mit dieser Forschungsstätte und mit ihrem Leiter in herzlicher Anhänglichkeit zu verbinden. Das ist die Wirkung jener ganz besonderen Forscheratmosphäre, jenes flotten, aufgeschlossenen, kameradschaftlichen Geistes, der getragen ist von der Persönlichkeit des geistigen Vaters dieser Anstalt: Franz RuTTNER.

Aus der Schule Molischs nahm er das solide Rüstzeug des Pflanzenphysiologen, die kritische Einstellung zu den neuen Problemen und den erfinderischen Geist mit an die von WOLTERECK organisierte biologische Anstalt, die Franz RUTTNER zu dem zu machen verstand, was sie jahrzehntelang war und auch heute - nach den Fährnissen und den Nachwirkungen des Zweiten Weltkrieges - wiederum ist: eine lebendige Forschungsstätte und ein Zentrum hydrobiologischer Forschung, das in der Welt einen ausgezeichneten Ruf erworben und behalten hat.

Franz RUTTNERS vielgestaltiges wissenscbaftliches Werk ist yornehmlich nach der theoretischen Seite hin ausgerichtet. In seinen ersten Arbeiten, die ins erste und zweite Dezennium unseres Jahrhunderts fallen, befasst er sich mit Fragen der Ökologie der Planktonorganismen, ihren täglichen Wanderungen und ihrem Verhalten gegenüber dem Lichtfaktor. Er schafft neue Methoden zur Erfassung und zum Studium des Zwerg- (Zentrifugen-) Planktons. Dann wendet er sich dem Lebensraum, den ein See darstellt, $\mathrm{zu}$, der Verteilung des Lichtes in verschiedenen Wassertiefen, der thermisch bedingten Stabilität, dem Kohlensäure-Kalk-Gleichgewicht und den Wechselwirkungen zwischen diesen Haushalten einerseits und der physio- 
logischen Tätigkeit der Mikro- und Makroorganismen eines Sees anderseits. Dabei bilden neben den Lunzer Seen (Ober-, Mittel- und Untersee) eine ganze Reihe österreichischer Gewässer den Gegenstand seiner Untersuchungen. Aber auch Fragen der Fischerei und des Abwassers finden in ihm einen zuverlässigen Bearbeiter.

War bis in die zwanziger Jahre hinein die Limnologie - ein Kind europäischer Wissenschaft - in weitgehendem Ausmasse auf diẹ Verhältnisse der gemässigten Zonen der Erde ausgerichtet, so erfuhr sie durch die deutsche Sunda-Expedition der Herren THIENEMANN, RUTTNER und FEUERBORN (1929/30) eine sehr bedeutsame Ausweitung auf die Binnengewässer tropischer Gebiete, über deren besonderen chemisch-physikalischen und biologischen Haushalt bis dahin recht wenig bekannt war. Der programmmässige Ablauf und die uiberaus reiche Ausbeute, die diese Expedition zutage förderte, lassen erkennen, mit welcher Zielsicherheit und Sachkenntnis sie vorbereitet und durchgefuihrt wurde. Ein sehr wesentliches Verdienst am guten Gelingen dieser bedeutenden Unternehmung darf gewiss dem ausgesprochenen Organisationstalent FR.ANZ RUTTNERs zugeschrieben werden.

Nicht geringere Bedeutung als seiner Forschung kommt RutTNERS Lebrtätigkeit zu. Von der Universität Wien zum Professor der Hydrobiologie ernannt, führte er bis heute alljährlich biologische Kurse für Studierende der Naturwissenschaften und Lehrer in Lunz durch und trug durch seinen klaren, lebendigen Unterricht viel wissenschaftliche Anregung in die Welt hinaus. Aber noch wirksamer wurde seine Lehrtätigkeit untermauert durch die Herausgabe seines Grundrisses der Limnologie, jenes kleinen, aber hochbedeutsamen Werkes, das kürzlich in zweiter Auflage und gleichzeitig in deutscher und englischer Sprache erschienen ist. Es stellt eine Fundgrube wertvoller, origineller Gedanken dar und enthält, auf engen Raum zusammengepackt und kritisch, klar und verständlich dargestellt, einen reichen Born an Dokumentationsmaterial, so dass jeder, der mit limnologischen Problemen arbeitet und in Hydrobiologie unterrichtet, immer wieder nach diesem vorbildlichein Buch greifen muss.

In Fachkreisen weiss man, was man Franz RuTTNER zu danken hat. Dieser Dank fand erfreulicherweise seinen Ausdruck in der Verleihung der Einar-Naumann-Medaille (I942) der Internationalen Vereinigung für Limnologie und durch die Ernennung zum Mitglied der Leopoldinischen Akademie der Naturforscher in Halle.

Möge dem noch jugendlich rüstigen Jubilar eine weitere Reihe von guten Jahren der Gesundheit und des Wohlergehens und der schöpferi- 
schen Arbeit beschieden sein, zum Besten der limnologischen Wissenschaft und aller weiteren Arbeitsgebiete, denen FrANz RUTTNER im Laufe eines reichen Lebens sein Interesse widmete.

Otto fag

In wissenschaftlichen Beiträgen zu einer

«Festschrift Franz Ruttwer»

hat eine grosse Zahl von Kollegen aus aller Welt dem Jubilar die persönliche Hochachtung und den Dank dargebracht. Die Drucklegung dieser Arbeiten in einem Sammelbande begegnete aber bedeutenden Schwierigkeiten. Als darum von befreundeter Seite her das Gesuch an uns erging, einen Teil der eingereichten Manuskripte zur Veröffentlichung in unserer Zeitschrift zu übernehmen, haben wir uns hiezu gerne bereit erklärt, um damit die guten Wünsche, die die schweizerischen Hydrobiologen an Herrn Prof. RU'T'TNER gerichtet hatten, durch die helfende Tat zu unterstreichen. Bisher sind uns in diesem Zusammenhange die Arbeiten der Herren DEVIDí, SCHILLER und BOURRELLY zugegangen. 